\title{
Experiencias
}

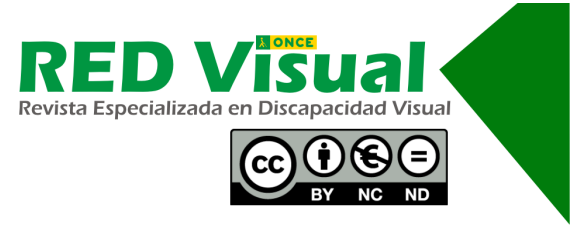

\section{Nosotros también participamos: talleres de autonomía y bienestar en residencias de la tercera edad ${ }^{1}$}

\author{
We can participate, too: independence and wellbeing workshops \\ in homes for seniors
}

\section{Senís Gilmartín, F. Sánchez Casado}

\section{Resumen}

Este trabajo nace de la preocupación de un psicólogo de la Residencia Las Cármenes, de Poblete (Ciudad Real), cuando observa que los usuarios con discapacidad visual participan muy poco de las actividades de bienestar y de ocio de la propia residencia. Se pone en contacto con la ONCE y, a partir de ahí, junto con el técnico de rehabilitación, diseñan un programa para estas personas con actividades de autonomía, ocio y entretenimiento con el objetivo de impulsar la integración de este colectivo en el día a día de la residencia. Este proyecto refleja el trabajo de 6 meses en la residencia con usuarios con discapacidad visual. En él se describen los objetivos propuestos, la metodología, la temporalización, los resultados y las conclusiones, si bien se ha de tener en cuenta, como en todo trabajo realizado con personas, que se han ido adaptando los objetivos y la propia metodología a estas. Señalar la parte positiva de los resultados, no solo los derivados de los talleres propuestos, y en cierta medida esperados, sino también otros producidos por la interrelación de los participantes, muy enriquecedores e inesperados.

\section{Palabras clave}

Autonomía. Autoestima. Capacidad. Relaciones sociales. Entretenimiento. Aprendizaje.

1 Trabajo galardonado con uno de los accésits del IV Concurso de Experiencias de Innovación y Buenas Prácticas en Servicios Sociales de la ONCE, presentado con el título Nosotros también participamos.

Senís, M., y Sánchez, F. (2020). Nosotros también participamos: talleres de autonomía y bienestar en residencias de la tercera edad. RED Visual: Revista Especializada en Discapacidad Visual, 76, 75-90. https://doi.org/10.53094/TAKC6725. 


\begin{abstract}
This experience was the outcome of the concern expressed by a psychologist at Residencia Las Cármenes in Poblete, a city in the Spanish province of Ciudad Real, who observed a low rate of participation in the home's wellbeing and leisure time activities among residents with visual disability. After consulting ONCE, he and one of the organisation's rehabilitation workers designed a programme for those people, which included activities geared to independence, leisure and amusement as a way of furthering their integration in the home's everyday life. The article describes the 6-month project, which targeted home residents with visual disability, with its objectives, methodology, timing, results and conclusions. As in all any activity involving people, however, the aims and methodology had to be adapted to participants. The results were highly satisfactory, not only the ones more or less expected for the workshops per se, but others not initially envisaged but very rewarding, such as the generation of participant inter-relationships.
\end{abstract}

\title{
Key words
}

Independence. Self-esteem. Aptitude. Social relations. Amusement. Learning.

C. es una mujer de 87 años decidida e independiente con unas ganas enormes de seguir cumpliendo etapas en el camino que va ofertándole la vida. Si echa la vista hacia atrás, se siente satisfecha, y si mira hacia delante, se siente con fuerzas de seguir aprendiendo.

Es valiente, y ha tomado la decisión de irse a una residencia de personas mayores. Su razón más contundente vino cuando en uno de esos folios de hospital le escribieron la palabra glaucoma. Empezó a sentir que su campo visual se estrechaba fruto (eso le dijeron) del incremento de la presión intraocular. Ella se decía una y otra vez: «Hay que estar preparada para los cambios de la vejez y no por ello hay que deprimirse, hay que buscar alternativas». Y así fue como la teoría la llevó a la práctica.

Cuando su hogar no tenía los mismos colores que antes, cuando la calle por la que tantas veces había caminado se hacía cada vez más cuesta arriba y los sonidos eran más estridentes, había que tomar una decisión sin titubear. C. era muy sincera consigo misma, y la dificultad visual fue el detonante para decidir que ya no podía vivir sola.

Senís, M., y Sánchez, F. (2020). Nosotros también participamos: talleres de autonomía y bienestar en residencias de la tercera edad. RED Visual: Revista Especializada en Discapacidad Visual, 76, 75-90. https://doi.org/10.53094/TAKC6725. 
Sopesó junto a su familia las alternativas, siempre partiendo de la misma hipótesis: la visión y los años no iban a poder dejarle fuera de una vida plena.

Muchas opciones eran acertadas, pero en su mente eran algo grises para aceptarlas sin miramientos. Hasta que una tarde de domingo llamó a su puerta uno de sus nietos:

-Abuela, he encontrado un sitio donde puedes vivir con todas las comodidades y atenciones que necesitas.

-Nieto, ya tengo una lista de residencias, pero hay algo que no termina de convencerme.

-Estoy seguro que esta residencia te sorprenderá. Tienen un grupo especializado para atender a usuarios con visibilidad reducida, hacen actividades, talleres y charlas para seguir manteniendo el nivel cognitivo. He hablado con una de las personas que van a ese grupo y me ha dicho que incluso está aprendiendo cosas que hasta ahora no conocía. iFíjate, abuela, a tu edad puedes seguir aprendiendo y conocer gente!

Y ahí, de repente, entre el color gris apareció un arco iris con todos los colores que necesitaba para seguir construyendo otra etapa de su camino vital.

C. es feliz, y tiene tantos alicientes positivos que se despierta todas las mañanas con ganas de sonreír. Es cierto que su cuerpo no es el de antes, pero ella conserva la misma vitalidad que años atrás.

\section{Presentación y justificación}

Si hacemos un estudio de las características actuales de la población de los afiliados a la ONCE, enseguida nos damos cuenta de que cada vez son más las personas mayores. En 2018, el 45,4\% de los afiliados tienen más de 65 años, siendo el desglose de esta manera: el $16,1 \%$ corresponde a personas de 65 a 74 años, el $15,8 \%$ son personas de 75 a 84 años y el 13,5\% tienen más de 85 años. Este envejecimiento de la población se traduce en que los profesionales de los equipos de atención de los Servicios Sociales de la ONCE se dirijan cada vez más a los mayores.

En este sentido, los programas de rehabilitación se desarrollan muchas veces en ámbitos diferentes a los domicilios particulares de cada uno. Es muy frecuente que la persona mayor pase su último periodo de vida en una residencia. En este caso, el éxito del programa ya no depende tanto de la motivación e interés del afiliado, sino

Senís, M., y Sánchez, F. (2020). Nosotros también participamos: talleres de autonomía y bienestar en residencias de la tercera edad. RED Visual: Revista Especializada en Discapacidad Visual, 76, 75-90. https://doi.org/10.53094/TAKC6725. 
también de la filosofía de la institución en la que reside, así como de la actitud del personal frente a la discapacidad visual.

Las necesidades vitales de las personas mayores que viven en residencias están cubiertas. Si la persona con discapacidad visual no es autónoma o ha dejado de serlo, el personal de la institución se encarga de realizar todas las tareas de higiene, cuidado personal, vestido, comida e incluso movilidad. Hay afiliados que, por distintas circunstancias, dejan cuidarse y ceden -o les obligan a ceder- su autonomía, llegando a producirse casos de sobreprotección; otros, sin embargo, siguen queriendo ser autónomos, y no dejan que hagan por ellos tareas que pueden hacer. En estos casos el trabajo del técnico de rehabilitación es más concreto y más efectivo, de ahí la importancia de motivar y empoderar a las personas para que mantengan o recuperen su autonomía.

Pero ¿qué ocurre con respecto al ocio, a los talleres y las actividades dirigidas a los residentes para fomentar el entretenimiento, la estimulación cognitiva, el aprendizaje y, en definitiva, una mejora de calidad de vida? ¿Participan nuestros afiliados? ¿Se sienten integrados en la rutina de la residencia? Esto depende, una vez más, de la dirección, el interés y la formación que tenga el equipo que conforma la residencia. Se ha de partir de que la persona con discapacidad visual, en general, siempre quiere participar en las actividades, formando así parte del colectivo, pero, en ocasiones, se encuentra barreras para hacerlo.

El origen del proyecto Nosotros también participamos nace de la inquietud del psicólogo de la Residencia STS Las Cármenes, en Poblete (Ciudad Real), del Grupo Colisée España, que se encuentra con un grupo de personas con deficiencia visual y ceguera -algunos afiliados a la ONCE- y que percibe cómo se quedan excluidas de algunas actividades por esta discapacidad. Pide información y formación a la ONCE para poder, no solo incluir a estas personas en muchas de las actividades de la residencia, sino también para poder realizar otras específicas.

El técnico de rehabilitación de la ONCE ve en esta petición la posibilidad de trabajar la autonomía con unos apoyos importantísimos, como es la buena disposición hacia el aprendizaje de los usuarios - ya que se trabajará de forma grupal, creándose muy buen ambiente-, la implicación del personal de la residencia y una consolidación de los objetivos, porque se incluyen en la cotidianidad de la misma. Gracias al trabajo en grupo se fomentan, además de la motivación por el

Senís, M., y Sánchez, F. (2020). Nosotros también participamos: talleres de autonomía y bienestar en residencias de la tercera edad. RED Visual: Revista Especializada en Discapacidad Visual, 76, 75-90. https://doi.org/10.53094/TAKC6725. 
aprendizaje, la integración y socialización de sus componentes, por lo que, de una manera genérica, engloba una forma de afianzar valores esenciales en esta etapa de la vida.

En una residencia, las actividades lúdicas que estimulen las funciones motoras, la actividad cognitiva y la participación social, se han convertido en una parte esencial de la programación que se ha de ofertar para mejorar la calidad de vida de nuestros mayores. Se trata de encontrar actividades que conecten con sus preferencias, para lograr su motivación y estimulación, y conquistar así su iniciativa de participar. Es una manera de fomentar que cada día se levanten con una ilusión de hacer, de compartir y de mejorar en las actividades que realizan.

Ahí entra el trabajo a realizar, brindando la posibilidad de hacer ese sueño realidad a todas esas personas que siguen teniendo inquietudes y que, por su falta de visión, quedan mermadas de actividades que pueden ser beneficiosas, pero que, al no estar adaptadas, no pueden formar parte de ellas de una manera activa.

\section{Objetivos}

\subsection{Objetivos curriculares}

El objetivo principal del proyecto es que personas con discapacidad visual en residencias puedan disfrutar plenamente de actividades y/o talleres de ocio, cultura y autonomía.

Para ello, es necesario también formar al personal de la residencia sobre cómo adaptar talleres, actividades y materiales para trabajar con personas que tienen esta discapacidad.

Los objetivos específicos de trabajo son los siguientes:

- Conocer los juegos adaptados de mesa. Ser capaces de jugar de forma autónoma con material en macrotipos y/o adaptados táctilmente.

- Reconocer monedas y billetes. Ser capaces de distinguir, por medio del tacto, las distintas monedas y los billetes de euro.

Senís, M., y Sánchez, F. (2020). Nosotros también participamos: talleres de autonomía y bienestar en residencias de la tercera edad. RED Visual: Revista Especializada en Discapacidad Visual, 76, 75-90. https://doi.org/10.53094/TAKC6725. 
- Conocer las posibilidades del sonido. Poder disfrutar de una lectura y/o de una película solo con el sonido, así como de conocer y saber manejar material específico para personas con discapacidad visual.

- Saber marcar material para su reconocimiento inmediato. La importancia del orden y la organización.

- Adiestrar y mejorar el comportamiento en la mesa. Conseguir comer de forma autónoma y activa en la mesa.

Junto a estos objetivos específicos hay otros implícitos en el desarrollo de los talleres, como es el mejorar la calidad de los usuarios en distintos ámbitos -como pueden ser la organización, la orientación espacial, la exploración de material, el tacto directo e indirecto, la memoria, la estimulación cognitiva, el aprovechamiento del resto visual, etc.-, a la vez que, por tratarse de talleres en grupo, se fomentan la iniciativa, la motivación, la socialización, la ayuda y también, a la vez, la competitividad.

\subsection{Objetivos cognitivos y conductuales}

El objetivo principal es detectar necesidades y solventarlas para favorecer la calidad de vida de los mayores que formen parte de nuestro proyecto. Este tipo de intervenciones se dirigen a optimizar la cognición, mejorar el estado de ánimo y la conducta, así como la funcionalidad de los pacientes con déficit visual.

A partir de este objetivo, desglosaremos otros más específicos:

- Estimular y mantener las capacidades cognitivas.

- Evitar la desconexión con el entorno y con sus iguales.

- Mejorar la ejecución de las actividades de la vida diaria.

- Mejorar la autoestima por medio de la participación y de las relaciones sociales.

- Promover el aprendizaje, la comunicación y la expresión.

\section{Población destinataria}

La población a la que va dirigido este proyecto es a personas que tienen una discapacidad visual y que viven en la Residencia STS Las Cármenes del grupo Colisée

Senís, M., y Sánchez, F. (2020). Nosotros también participamos: talleres de autonomía y bienestar en residencias de la tercera edad. RED Visual: Revista Especializada en Discapacidad Visual, 76, 75-90. https://doi.org/10.53094/TAKC6725. 
España, ubicada en Poblete, en la provincia de Ciudad Real, si bien podría ser aplicado e implantado en residencias donde hubiera un número de afiliados que pudieran beneficiarse de su dinámica. Personas con baja visión, que no están afiliadas a la ONCE porque no llegan al baremo necesario, pueden beneficiarse también de las actividades y talleres del proyecto.

Las características visuales de cada participante no son importantes o determinantes, ya que la metodología del trabajo a realizar se puede adaptar a todo el abanico de la discapacidad visual, es decir, desde un buen resto visual muy funcional hasta la ceguera total. Si las personas que participan en el proyecto tienen otras discapacidades, estas no tienen por qué ser excluyentes, ya que se hará lo posible para su inclusión en las tareas y actividades.

Es importante que la población que participe en el proyecto tenga un nivel cognitivo suficiente para poder seguir de forma activa las explicaciones e indicaciones, y que puedan comprender el alcance de cada actividad.

La posibilidad de que en los grupos haya alguna persona sin discapacidad visual que ayude al buen funcionamiento del proyecto se contempla como algo bueno y necesario para el desarrollo del mismo. Nos referimos a personas activas, con cierta capacidad de liderazgo y con predisposición de ayuda para sus compañeros.

El presente proyecto es de carácter correlacional, ya que pretende establecer relaciones entre los resultados obtenidos, con la finalidad de obtener los objetivos propuestos.

En un principio, se instaura un grupo piloto dentro de la Residencia STS Las Cármenes que sirva de precedente y pueda seguir creciendo en número de participantes, así como en difusión. Este grupo inicial está formado por 7 mujeres pertenecientes a la residencia. Todas las participantes tienen en común el padecimiento de algún tipo de déficit visual y muchas ganas de aprender y de vivir experiencias.

Es de señalar que la finalidad de este proyecto es que llegue a más gente y puedan participar todas las personas que lo necesiten, por lo que puede que la población varíe según se avance, ya que puede haber ingresos nuevos en la residencia que cumplan con los requisitos para participar.

Senís, M., y Sánchez, F. (2020). Nosotros también participamos: talleres de autonomía y bienestar en residencias de la tercera edad. RED Visual: Revista Especializada en Discapacidad Visual, 76, 75-90. https://doi.org/10.53094/TAKC6725. 


\section{Metodología}

Se ha llevado a cabo una metodología activa, mediante un grupo reducido (no más de 10 personas) para poder trabajar mejor en equipo. Se utilizaron las instalaciones de una residencia para la realización de las actividades propuestas, en particular la sala de biblioteca.

Las actividades son grupales, puesto que van dirigidas a un mismo colectivo en común, si bien deben tener en cuenta las capacidades individuales para así adaptarlas más consecuentemente según la ayuda que puedan necesitar.

El técnico de rehabilitación de la ONCE prepara la actividad conjuntamente con el psicólogo de la residencia, bien llevando material específico para personas con discapacidad visual, o bien utilizando el material existente en ella susceptible de adaptación o uso común. Cada actividad se repetirá en varias ocasiones, estando en la primera sesión el técnico de rehabilitación como experto en la materia, pudiéndose realizar la actividad las veces que se considere oportuno para afianzar los aprendizajes sin la presencia del técnico de rehabilitación y contando con los distintos profesionales de la residencia que estén interesados en el proyecto y colaboren con el mismo (psicólogo, terapeuta ocupacional, animador sociocultural, educador social...).

El tiempo estimado de cada sesión es de una hora y media aproximadamente. Cada taller, por tratarse de aprendizajes específicos y diferentes tendrá su propia identidad y material, aunque podemos hacer una generalización en la metodología:

- Presentación de la actividad.

- Presentación de los distintos materiales, identificación de los mismos y sus características.

- Explicaciones y prácticas de pequeñas tareas para consolidar el material y su funcionamiento.

- Actividades de interrelación entre los componentes del grupo.

- Juegos de consolidación de objetivos.

Senís, M., y Sánchez, F. (2020). Nosotros también participamos: talleres de autonomía y bienestar en residencias de la tercera edad. RED Visual: Revista Especializada en Discapacidad Visual, 76, 75-90. https://doi.org/10.53094/TAKC6725. 
- Reflexión sobre el taller.

Se considera necesaria una pequeña formación sobre la discapacidad visual para el personal de la residencia y, en especial, para los que pudieran participar en el proyecto. La formación la llevaría a cabo el técnico de rehabilitación y sería aproximadamente de dos horas.

Los talleres elegidos para el proyecto son los siguientes:

- Taller 1: Juegos de mesa adaptados. Se presentaron juegos adaptados para las personas con discapacidad visual (ciegas y con deficiencia visual), para demostrar que se podía jugar sin ver, y, a continuación, se hicieron juegos en grupo y/o por parejas. Los juegos utilizados fueron el parchís, las cartas macrotipo, las tres en raya, el dominó y el bingo.

- Taller 2: Identificación del dinero. Se describieron todas las monedas una por una, indicando las características táctiles que se pueden apreciar para identificarlas sin ver. Se hizo lo mismo con los billetes. Se habló de la importancia de la organización de monedas y billetes.

- Taller 3: Material específico. Se realizaron actividades con materiales específicos que permiten aumentar la autonomía en algunas actividades de la vida cotidiana, materiales como el reloj parlante y el reloj macrotipo, agujas de ciego y material de costura, entre otros.

- Taller 4: Organización y marcaje. Se escogieron materiales cotidianos y muy necesarios para la vida diaria, y se hicieron prácticas de cómo marcarlos (marcaje visual, marcaje táctil) y ordenarlos para su identificación y manipulación.

- Taller 5: Adiestramiento en la mesa. Se realizaron actividades para comer de forma adecuada sin ver, utilizando trucos de orientación en la mesa y de abordaje de platos, cubiertos y vasos.

- Taller 6: El sonido como alternativa. Se dieron a conocer los libros en audio, el sistema audesc, el teléfono móvil (parlante y con ampliaciones) y otras posibilidades de acceder a la información a través del sonido. Se realizaron actividades también para el manejo de estos dispositivos, para que comprobaran su fácil manipulación.

Senís, M., y Sánchez, F. (2020). Nosotros también participamos: talleres de autonomía y bienestar en residencias de la tercera edad. RED Visual: Revista Especializada en Discapacidad Visual, 76, 75-90. https://doi.org/10.53094/TAKC6725. 


\section{Temporalización}

Se ha llevado a cabo de manera quincenal, desde el mes de enero hasta el mes de julio de 2019. El técnico de rehabilitación ha estado presente en la primera quincena o en la primera sesión de cada taller. La sesión o sesiones de repaso se dieron en la otra quincena, desarrollándolas el psicólogo y otros colaboradores de la residencia.

La duración de las sesiones era de 90 minutos. En nuestro caso, el horario era desde las 10.30 horas a 12.00 horas. El día de la semana dependía de los profesionales y de las instalaciones de la residencia.

\section{Resultados}

Los resultados, en general, son positivos. Se irán analizando taller por taller, ya que cada uno tiene unas peculiaridades muy concretas y definidas $y$, en muchas ocasiones, dispares.

\section{Taller 1: Juegos de mesa adaptados}

El resultado es positivo en cuanto al entretenimiento de los usuarios, a la práctica del sentido del tacto, la comprensión de los distintos materiales que componen cada juego y la identificación de los mismos.

Así mismo, el resultado fue muy positivo frente a las expectativas de juego y la posibilidad de poder jugar sin ver. Muchas personas mayores dan por hecho que «no ven», porque su visión es tan defectuosa que no llegan a reconocer los dibujos y detalles (en este caso, cada carta). Cuando les presentamos los macrotipos, es decir, cartas muy grandes en tamaño y con las figuras contrastadas con el fondo, sí eran capaces estas personas de distinguir cada carta.

En el desarrollo del taller se ha encontrado cierta dificultad a la hora de realizar un juego completo entre todos los componentes del grupo por la disparidad cognitiva y de actitud de cada participante frente al juego. Es por ello que consideramos que, para que este taller funcione, los juegos elegidos deben de ser sencillos y, sobre todo, de pocos participantes; por lo menos, al principio. Las tres en raya es un buen ejemplo de juego a utilizar inicialmente.

Senís, M., y Sánchez, F. (2020). Nosotros también participamos: talleres de autonomía y bienestar en residencias de la tercera edad. RED Visual: Revista Especializada en Discapacidad Visual, 76, 75-90. https://doi.org/10.53094/TAKC6725. 


\section{Taller 2: Identificación del dinero}

El resultado es positivo en cuanto a que los participantes descubrieron la posibilidad de controlar e identificar el dinero sin ver.

En el desarrollo del taller se hizo evidente una gran disparidad entre los participantes según sus capacidades cognitivas y de memoria. Había usuarios que no solo les costaba más tiempo el reconocimiento, sino que también necesitaban que se les introdujeran monedas nuevas de forma más espaciada y dosificada.

Las personas mayores en las residencias están tan acostumbradas a que se lo hagan todo que no tocaban apenas los objetos que les presentábamos. Pero, una vez les decías que lo hicieran con las dos manos y que exploraran adecuadamente, notaban todas las características y matices de los objetos, de tal forma que podían identificarlos y manipularlos. Por tanto, en general, mostraban muy buena capacidad para el tacto, aunque era necesario recordarles técnicas de exploración constantemente.

Aunque es un taller de poca utilidad en el sentido práctico (se hace poco uso del dinero en la residencia), los usuarios se mostraron muy satisfechos. El poder saber cuánto dinero tienen en un momento determinado, cosa tan importante a lo largo de tu vida, fue como recuperar una capacidad que siempre habían tenido y que habían perdido, haciéndoles más partícipes de su propia vida.

\section{Taller 3: Material específico}

Se obtuvo un resultado muy positivo en el uso de materiales concretos, de manejo fácil y de gran utilidad, como son el reloj parlante, el reloj de alto contraste, la aguja de ciego y el avisador de luz. Las personas mayores se sienten muy motivadas por tareas sencillas que no les complique mucho la vida y que tengan un resultado inmediato, como es apretar un botón y saber la hora.

Otros materiales específicos, como el organizador de calcetines y el avisador de líquidos o el metro de costura adaptado, también gustaron, si bien no tienen una utilidad práctica en la residencia. Estos materiales son de fácil manejo, pero en las residencias les lavan la ropa, les cosen las prendas rotas o deterioradas y sirven rápidamente el agua. No descartamos, sin embargo, que, para ciertas personas - usuarios y personal de la residencia- podría ser un punto de partida

Senís, M., y Sánchez, F. (2020). Nosotros también participamos: talleres de autonomía y bienestar en residencias de la tercera edad. RED Visual: Revista Especializada en Discapacidad Visual, 76, 75-90. https://doi.org/10.53094/TAKC6725. 
para cambiar estos hábitos y que dichas tareas las hagan los residentes, aunque vean poco o nada.

Como se pudo comprobar en el desarrollo de este taller, el poder controlar la hora y ser un poco más autónomos en ciertas tareas cotidianas hacen que los usuarios sean más partícipes en la vida de la residencia y, por tanto, más autónomos e independientes.

\section{Taller 4: Organización y marcaje}

Resultado positivo en cuanto a la comprensión de la utilidad del marcaje, tanto para la identificación de materiales y objetos como para hacer más fácil su manejo. Se explicó el marcaje por organización, por características físicas de los objetos; en vista, por marcas contrastadas, y por tacto, con marcas táctiles. Estas últimas fueron las que más gustaron por su sencillez y fácil localización (gotas de silicona).

Pese a que encontramos que, al igual que otras de las actividades mencionadas, no tienen un desarrollo práctico en su día a día, ya que no tienen gran necesidad de marcar objetos y utensilios, todos los usuarios fueron conscientes de la gran utilidad que tiene el marcaje en una vivienda propia para poder seguir siendo autónomos (lavadora, plancha, cocina, etc.). Dentro de este taller se obtuvieron resultados satisfactorios para los participantes, ya que, en su propio espacio personal, se reorganizaron algunos marcajes, como, por ejemplo, interruptores de la luz, que, gracias a las gotas de silicona, les supuso una mejora en el día a día.

\section{Taller 5: Adiestramiento en la mesa}

Como resultado positivo, podemos afirmar que tanto el repaso de métodos de orientación en la mesa, así como la exploración de alimentos en el plato, como su manipulación, fue muy agradable e instructivo para los participantes, que aprendieron a manejar pautas facilitadoras de tal tarea. Finalidad emergente hacia una nueva actitud más activa del propio comportamiento en la mesa. Destacar la sorpresa ante algunas personas con visibilidad nula que lograron cortar un filete de la manera más óptima.

A la hora de llevar a cabo el taller, se ha de ser consciente de la gran disparidad en el uso de cubiertos entre los usuarios dependiendo de sus costumbres y hábitos, y no de su discapacidad visual. Así mismo, se ha de ser consciente de la barrera que

Senís, M., y Sánchez, F. (2020). Nosotros también participamos: talleres de autonomía y bienestar en residencias de la tercera edad. RED Visual: Revista Especializada en Discapacidad Visual, 76, 75-90. https://doi.org/10.53094/TAKC6725. 
supone la propia actividad normal de la residencia en los comedores, que debe ser rápida y efectiva, y que provoca en los cuidadores una actitud de sobreprotección hacia las personas con discapacidad visual muy grande y difícil de cambiar. Para ello, el usuario debería ser muy eficaz en las tareas de cortar y servirse líquidos. Y para que esto suceda, haría falta un entrenamiento diario con supervisión.

\section{Taller 6: El sonido como alternativa}

Según la fuente de sonido, los resultados fueron muy diferentes. En cuanto a las películas en audesc, solo tuvieron un resultado positivo con los usuarios acostumbrados al sonido como fuente de información; es decir, los participantes que utilizan de forma cotidiana la TV y la radio. Al resto de los usuarios les costaba centrarse en el audio y comprender el contenido.

En cuanto a los libros en audio es una posibilidad de lectura en sonido estupenda para los lectores de toda la vida, aunque dudan a la hora de comprar el reproductor por el precio. Todos somos conscientes del excelente resultado de este dispositivo para leer, pero está altamente relacionado con los intereses personales de cada lector.

Si bien se planteó la posibilidad de incorporar un teléfono móvil con voz en el taller, la misma fue rechazada por los usuarios, ya que les parecía complicado y, en muchos de los casos, un gasto excesivo, por lo que se decidió no incorporar este dispositivo a la dinámica del taller.

Como resultado positivo, podemos afirmar que el taller sirvió para consolidar e impulsar aquellas actividades que se pueden adaptar con y por sonido (lectura de cuentos, talleres de relajación, talleres de poesía, análisis de textos, etc.), tanto por parte del personal de la residencia como por parte de los usuarios.

\section{Conclusiones}

Según la Organización Mundial de la Salud (OMS), el bienestar emocional es un «estado de ánimo en el cual la persona se da cuenta de sus propias aptitudes, puede afrontar las presiones normales de la vida, puede trabajar productiva y fructíferamente y es capaz de hacer una contribución a la comunidad». En una visión más amplia, también se habla de bienestar emocional en relación a la capacidad de adaptación a

Senís, M., y Sánchez, F. (2020). Nosotros también participamos: talleres de autonomía y bienestar en residencias de la tercera edad. RED Visual: Revista Especializada en Discapacidad Visual, 76, 75-90. https://doi.org/10.53094/TAKC6725. 
los cambios, la flexibilidad para aprender cosas nuevas, así como tener sentido del humor, conjuntamente con un estilo de vida saludable y activo.

Lo que es indiscutible es que cada uno de los participantes, dentro de sus características individuales, ha ido experimentado cierto bienestar emocional que le ha animado a seguir en el proyecto con una motivación patente en el entusiasmo con el que han participado.

La experiencia en este tipo de actividades ha permitido poner de manifiesto en muchos de estos residentes funciones cognitivas y capacidades instrumentales que habían quedado olvidadas por el desuso dentro de un colectivo del que el déficit visual les ha privado de formar parte de manera activa. Su descubrimiento ofrece momentos de gran satisfacción, especialmente al propio sujeto, reforzando el grado de autoestima.

Las personas que presentan discapacidad visual siguen teniendo necesidades tales como la necesidad de sentir la valía personal, expresar los propios pensamientos y sentimientos, la necesidad de tener sentimiento de realización y de tener una rutina. Por lo que el principal problema adaptativo dentro de la residencia se basa en la incapacidad de estas personas para satisfacer las necesidades citadas anteriormente. Por ello, las actividades que hemos llevado a cabo son actividades que les resulta interesantes, estimulantes y retadoras, pudiendo ayudarles de esta forma a solventar esas necesidades.

Un concepto primordial que sale fuertemente beneficiado es la motivación, forjando a la persona como el elemento principal del proyecto. Por tanto, es muy importante que los retos propuestos sean personalizados, participativos y colaborativos. Tampoco se debe pasar por alto el fomento de entornos favorables basados en objetivos como el apoyo mutuo, la cohesión y las relaciones personales.

Seguidamente, cuando se fortalece la motivación, aparece el segundo concepto ligado a ella: la autoestima, a través del desarrollo de la sociabilidad y la inclusión en entornos favorables. En este sentido, se debe potenciar especialmente el sentimiento de utilidad. Proponer retos a los participantes, y que puedan comprobar por sí mismos que son capaces de llevarlos a cabo, les supone un gran valor añadido. Esta acción puede generar sentimientos de realización y orgullo, incluso proyectar nuevas ilusiones de futuro.

Senís, M., y Sánchez, F. (2020). Nosotros también participamos: talleres de autonomía y bienestar en residencias de la tercera edad. RED Visual: Revista Especializada en Discapacidad Visual, 76, 75-90. https://doi.org/10.53094/TAKC6725. 
Teniendo en cuenta la situación personal y las circunstancias que envuelven las vidas de muchos de los residentes, se comprende que, a veces, no es fácil sobrellevar esta etapa de la vida por la que están pasando. Son muchas las variables que pueden crear ansiedad y muchas las circunstancias que pueden llevar a un estado de ánimo depresivo. Otras veces, las circunstancias de cada uno pueden provocar episodios que lleva a los residentes a sentirse solos. Por lo que este proyecto, además de enseñar una manera de relacionarse con el entorno, de integrar una serie de actividades y de alcanzar algunas metas, también intenta hacer que los participantes se sientan integrados y motivados dentro de un grupo de iguales.

Junto a este apartado emocional-conductual, es importante resaltar otros aspectos extraídos de la experiencia y que permiten validarla:

- Los participantes de este proyecto acaban teniendo una actitud más activa ante la vida. El hecho de realizar las tareas de los distintos talleres, de forma independiente, sin esperar a que el auxiliar o cuidador de la residencia se lo haga, hace que se den cuenta de las capacidades dormidas u olvidadas que tienen con respecto a la autonomía, y se activa automáticamente el «yo puedo».

- Los talleres han permitido la recuperación de muchas actividades y el aprendizaje de otras que nunca habían realizado o de las que no habían sido partícipes, como son los juegos de mesa, la lectura, distintos dispositivos con sonido...

- A su vez, los talleres han posibilitado el descubrimiento del sonido y del tacto como fuentes de información, así como el aprendizaje de las capacidades táctiles y hápticas, de su entrenamiento y utilidad. La mayoría de los participantes están acostumbrados a que les den las cosas y se las hagan funcionar. Después del proyecto, su actitud es de tendencia a buscar con las dos manos, explorar e identificar de forma activa.

- Se han incorporado nuevas actividades en la residencia adaptadas en sonido. Asimismo, talleres de relajación, cuentacuentos o tertulias cobran un mayor protagonismo, y se insiste mucho en que participen todas las personas con discapacidad visual.

- Se ha concienciado a los usuarios del proyecto, tanto al personal de la residencia como a los propios participantes, de la importancia de los colores y del buen

Senís, M., y Sánchez, F. (2020). Nosotros también participamos: talleres de autonomía y bienestar en residencias de la tercera edad. RED Visual: Revista Especializada en Discapacidad Visual, 76, 75-90. https://doi.org/10.53094/TAKC6725. 
uso de los contrastes para todo tipo de actividades, materiales y estancias, ya que el contraste de colores entre los muebles, paredes, puertas, escaleras y otras dependencias y elementos ayudan a la localización y a la orientación de los residentes.

- Nuevas afiliaciones a la ONCE, que demuestran el interés creado por el proyecto y, en la mayoría de los casos, un conocimiento real de la funcionalidad de su visión. Nos ha llamado la atención el buen ajuste emocional que tenían los usuarios con respecto a su discapacidad visual.

- Se ha comprobado que en los talleres, pese a la disparidad de grados de visión, todos funcionaban igual; el peso de la consecución de los objetivos radica en las capacidades cognitivas.

- Como propuesta de mejora, se ha de señalar que los objetivos curriculares del proyecto se consolidarían mejor si se integraran en la cotidianidad del funcionamiento de la residencia, como es el caso de conseguir ser más autónomos en el comportamiento en la mesa. Otros ejemplos serían el uso del ascensor (marcando la botonadura), el uso de la máquina de bebidas (marcando los productos), la zona de juegos (con juegos adaptados y accesibilidad del entorno), etc.

Marta Senís Gilmartín. Técnico de rehabilitación de la ONCE en Ciudad Real. Delegación Territorial de la ONCE en Castilla-La Mancha. Calle Reino Unido, 10; 45005 Toledo (España). Correo electrónico: maseg@once.es.

Felipe Sánchez Casado. Psicólogo en la Residencia sTs Las Cármenes. Avenida de Ciudad Real, s/n; 13195 Poblete (Ciudad Real, España). Correo electrónico: felipesanchezcasado@gmail.com.

Senís, M., y Sánchez, F. (2020). Nosotros también participamos: talleres de autonomía y bienestar en residencias de la tercera edad. RED Visual: Revista Especializada en Discapacidad Visual, 76, 75-90. https://doi.org/10.53094/TAKC6725. 